\title{
Radio and X-ray observations of supernovae in dense environments
}

\author{
Poonam Chandra \\ National Centre for Radio Astrophysics, Tata Institute of Fundamental Research \\ Pune University Campus, Ganeshkhind, Pune 411 007, India \\ email: poonam@ncra.tifr.res.in
}

\begin{abstract}
Here we discuss the observational properties of supernovae exploding in extremely dense environments, namely Type IIn supernovae (SNe IIn). In SNe IIn, the surrounding environments play significant role in the supernovae energetics and evolution. Thus they are different than other classes of core collapse supernovae, whose energetics are not significantly altered by their environments. Though high density of medium is a prerequisite for radio and X-ray emission, less than $10 \%$ on SNe IIn are bright in these bands. This has important implications for their progenitor models. I will discuss the radio and X-ray observations of SNe IIn, which are crucial to unravel their complex environments. We also discuss some individual supernovae belonging to this class and discuss as to how they have refined our understanding of SNe IIn. Finally the importance of well sampled long term light curves in radio and X-ray bands cannot be stressed enough.
\end{abstract}

Keywords. supernovae: general, circumstellar matter, radiation mechanisms: general, supernovae: individual(SN 1995N, SN 2006jd, SN 2010j1)

\section{Introduction}

Massive stars (mass $\geqslant 8 M_{\odot}$ ) loose mass vigorously $\left(10^{-5}-10^{-6} \mathrm{M}_{\odot} \mathrm{yr}^{-1}\right.$ as compared to $\sim 10^{-14} \mathrm{M}_{\odot} \mathrm{yr}^{-1}$ in the Sun) from their outer layers towards the end stages of the evolution. Thus at the time of their demise as supernovae ( $\mathrm{SNe}$ ), they are surrounded by dense environments, created by the mass loss, termed as circumstellar medium (CSM). Usually the optical light curves and spectra in SNe are dominated by the explosion dynamics and the presence of the CSM has negligible effect. However, in a particular class of SNe, named Type IIn supernovae (SNe IIn), the mass loss is extremely high $\left(10^{-4}-10^{-1} \mathrm{M}_{\odot} \mathrm{yr}^{-1}\right)$, suggesting instability in the evolution of the progenitor just before the explosion. In these SNe, the CSM manifests itself in the form of narrow emission lines in their optical spectra. The bolometric and $\mathrm{H}-\alpha$ luminosities become quite high due to the contribution from the interaction of the supernova (SN) ejecta with the CSM (Chugai 1990). Due to this extra source of energy, they are detectable up to cosmological distances. The highest redshift Typs IIn SN is at a redshift of 2.36 (Cooke et al. 2009). SNe IIn are rare and they make less than $10 \%$ of the total core collapse SNe (Taddia et al. 2013).

In a SN explosion, fast moving ejecta colliding with the CSM leads to a blast wave 'forward' shock with a velocitiy $v \sim 10,000 \mathrm{~km} / \mathrm{s}$ and temperature $T \sim 10^{9} \mathrm{~K}$ and a 'reverse' shock propagating into the stellar envelope with $v \sim 1000 \mathrm{~km} / \mathrm{s}$ relative to the expanding stellar ejecta, heating it to $T \sim 10^{7} \mathrm{~K}$ (see Fig. 1). The heated shocked plasma in the forward and reverse shocks give rise to X-ray emission. Additionally, the accelerated relativistic electrons in the forward shocked shell in the presence of the amplified magnetic field give rise to radio emission (Chevalier \& Fransson 2016, Chevalier \& Fransson 2003). Since the shock velocities are typically 100-1000 times the speed of the progenitor wind, 


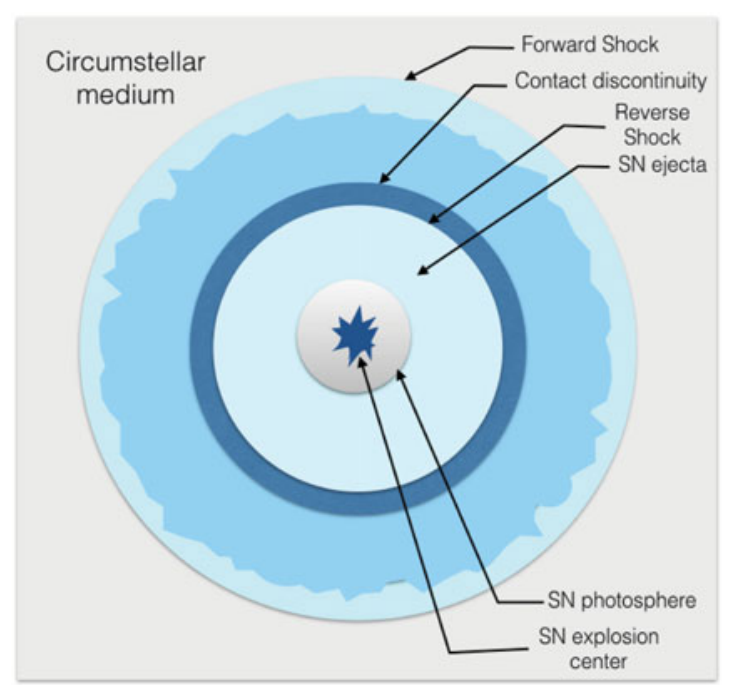

Figure 1. Schematic diagram of the ejecta interaction with the circumstellar medium. The diagram is not to scale.

the shock wave probes the past history of the star by sampling regions of the wind lost many hundreds-thousands of years ago.

In SNe IIn, the strong ejecta-CSM interaction leads to radiative shocks early on. In normal core collapse SNe, where the ejecta kinetic energy has only 1-2\% contribution to the total radiated energy, in SNe IIn up to $50 \%$ of the ejecta kinetic energy can go to the radiated energy (Smith 2016). In this article, we discuss the radio and X-ray emission from SNe IIn. In $\S 2$, we discuss the SNe IIn in general, their progenitor models and diversity. Our understanding of SNe IIn through radio and X-ray observations are discussed in $§ 3$. Here we also discuss some important SNe IIn which have contributed significantly to our understanding. In $\S 4$, we summarize the main results.

\section{Overview of SNe IIn}

SNe IIn are not an intrinsic class of SNe exploding from a particular kind of progenitor stars. The prerequisite for their classification is a dense H-rich material surrounding the progenitor star. The diversity of the environment plays crucial role in the evolution and come to dominate the energetics of SNe IIn, e.g., a weak explosion in a dense CSM may appear very bright (Smith 2016). For this reason SNe IIn have displayed great deal of diversity. For example, SN 1998S was initially classified as a bright Type IIL SN. However, at a very early stage ( $<10$ days) characteristics of a IIn SN emerged. This also shows connection between IIn and IIL SNe. While SN 1988Z and SN 1986J remained bright for a decade after explosion, SN 1994W faded in only 130 days. After the discovery of unusually bright SN 2006gy with an absolute magnitude of about -21.5 (Smith et al. 2007), a new category of superluminous supernovae (SLSNe) has been identified. All SLSNe II are essentially IIn, except one Type IIL case, suggesting dense environments may be crucial in these SNe. A special class of SNe IIn has shown spectral similarities with Type Ia thermonuclear SNe. Some of these events are SNe 2002ic and 2005gj. They are considered hybrid between SNe Ia and IIn. Some SNe IIn also seem to be related to $\mathrm{SNe} \mathrm{Ib} / \mathrm{c}$, e.g. SN $1995 \mathrm{~N}$ was most likely a Type Ib/c exploding in a dense wind. SN 2001em and SN 2014C are unique SNe. They were identified at maximum light as a 
$\mathrm{SN} \mathrm{Ib/c,} \mathrm{later} \mathrm{they} \mathrm{revealed} \mathrm{strong} \mathrm{interaction} \mathrm{with} \mathrm{a} \mathrm{dense} \mathrm{CSM} \mathrm{and} \mathrm{were} \mathrm{reclassified}$ as SNe IIn (Chugai \& Chevalier 2006, Milisavljevic et al. 2015).

While stellar models do not allow the kind of mass loss rates required to explain the $\mathrm{SNe}$ IIn, some theoretical stellar evolution models may incorporate enhanced mass loss shortly before explosion, these are super-asymptotic giant branch stars $\left(8-10 M_{\odot}\right)$, massive red supergiant stars $\left(17-25 M_{\odot}\right)$ and luminous blue variables $\left(\mathrm{LBVs} ;>35 M_{\odot}\right)$. LBV progenitors are more favorable models for bright SNe IIn. However, LBV is a transient phase between O-type and Wolf-Rayet (WR) star and stars are not supposed to explode in this phase. Even if they explode, the fine tuning of the explosion at the time of enhanced episodic mass loss is not understood. In addition, Anderson et al. (2012) using $\mathrm{H}-\alpha$ and near-UV data suggested that SN IIn progenitors come from low mass progenitors. Chevalier (2012) proposed a binary model where common envelope evolution can give rise to SNe IIn. In this model, the SN is triggered by the inspiral of a compact neutron star or black hole to the central core of the binary star.

However, archival data have suggested LBVs as progenitors in particular cases of Type IIn SN 2005gl (Gal-Yam et al. 2007) and SN 2010jl (Smith et al. 2011). In SN 2009ip, multiple pre-SN eruptions have been observed before the star exploding as SN IIn, strengthening the connection with LBV like progenitor (Mauerhan et al. 2013). Ofek et al. (2014) carried out analysis of Palomar Transient Factory archival data for more than $16 \mathrm{SNe}$ IIn and inferred that more than 50\% SNe IIn have precursors consistent with LBVs. In contrast, Bilinski et al. (2015) analysed 12 years of KAIT archival data at known SNe IIn locations and found no precusors, contradicting the above arguments. Recently Fox et al. (2017) presented HST/WFC3 imaging of the SN 2010jl field obtained in 2014, 2015, and 2016, where faint SN was seen 0.61" away from the blue source previously considered progenitor LBV star. Smith et al. (2016b) in late time HST observations found no trace of massive star formation at the SN 2009ip site. If LBVs are indeed transient phase between O-type and WR stars, they should be found in massive star forming regions. Smith \& Tombleson (2015) concluded that LBVs are not found in O-type clusters and are statistically more isolated than O-type and Wolf-Rayet stars. Based on the relatively isolated environments of LBVs, they have challenged the traditional association of the LBVs and suggested that LBVs are product of binary evolution. The explained LBVs to be evolved massive blue stragglers. Smith et al. (2016b) suggested that the progenitor of SN 2009ip too was the product of a merger or binary mass transfer.

\section{Radio and X-ray emission}

SN CSM is best probed by studying the signatures of the interaction of SN ejecta with the CSM. In SNe IIn with very high CSM densities, the ejecta CSM interaction is expected to emit copiously in radio and X-ray bands. However, the statistics is contradictory. While $\sim 400$ SNe IIn are known, only 12 are shown to emit in X-ray bands; these include SNe $1978 \mathrm{~K}, 1986 \mathrm{~J}, 1988 \mathrm{Z}, 1995 \mathrm{~N}, 1994 \mathrm{~W}, 1996 \mathrm{cr}, 1998 \mathrm{~S}, 2005 \mathrm{ip}, 2005 \mathrm{kd}, 2006 \mathrm{gy}, 2006 \mathrm{jd}$ and 2010jl (Ross \& Dwarkadas 2017). The statistics is not much better in radio bands either. Less than half SNe IIn $(\sim 154)$ have been looked in the radio bands (mainly with the Very Large Array (VLA)) and $\sim 10 \%$ have been detected.

However, there is a very interesting trend in radio and X-ray detected SNe IIn. While $\mathrm{X}$-ray luminosities are towards the high end in SNe IIn, the radio luminosities are diverse and occupy 4 orders of magnitude (Fig. 2). Radio light curves are extremely diverse, the diversity translate to diversity in the stellar mass loss and could indicate the different mass loss history. 


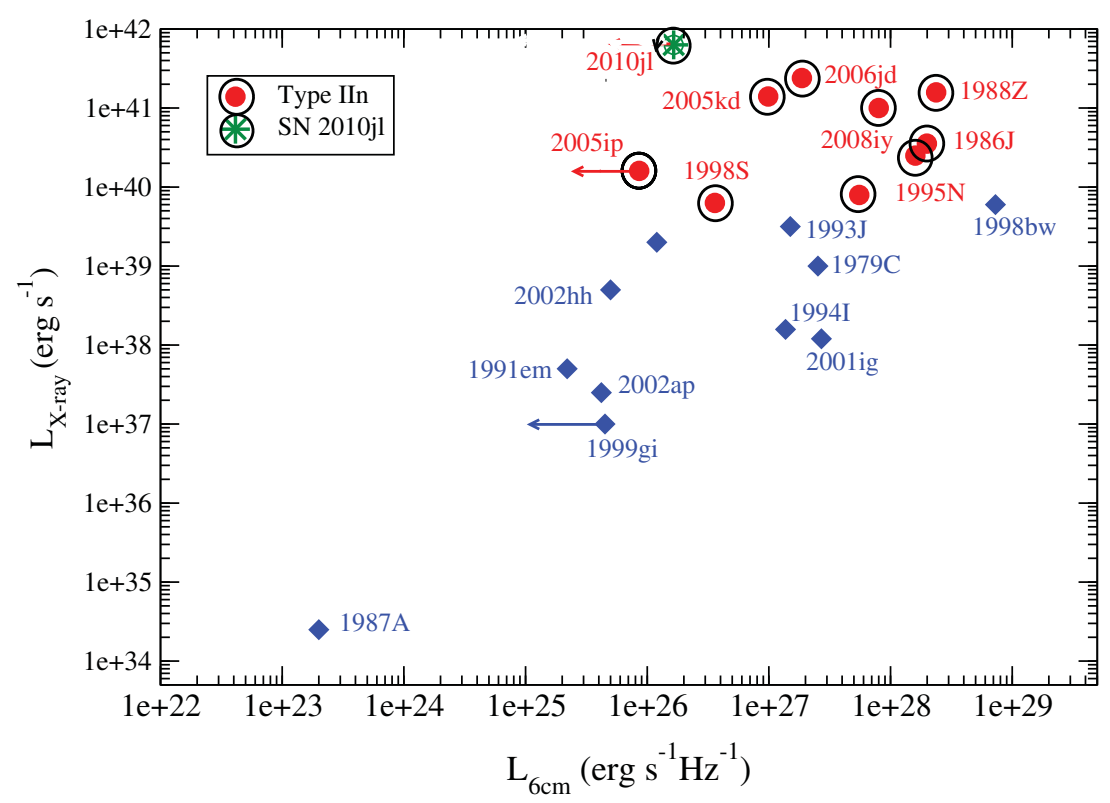

Figure 2. Peak radio and X-ray luminosities of some core collapse SNe. While radio luminosities occupy 4 orders of magnitude in SNe IIn and do not stand out from rest of the core collapse $\mathrm{SNe}$, their X-ray luminosities are amongst the highest.

\subsection{X-ray Luminosity evolution}

Since reverse shock has higher density, the thermal X-ray emission from core collapse SNe, in general, has dominating contribution from the reverse shock. In SNe IIn, a radiative cooling shell forms between the reverse and forward shock. The radiative cooled shell absorbs most of the X-rays emanating from the reverse shock (Nymark et al. (2006). Observations have also shown that the detectable contribution from SNe IIn mainly comes from the dense forward shock (Chandra et al. 2012, Chandra et al. 2015).

While the thermal X-ray luminosity is expected to decline as $\sim 1 / t$ for a steady wind, this relation is seen to be followed in hardly any core collapse SNe including SNe IIn (See 3 and (Dwarkadas \& Gruszko 2012, Dwarkadas et al. 2016). This could be because other mechanisms like inverse-Compton scattering etc. may be at play. There is high probability that wind any not be steady $\rho \propto 1 / R^{2}$ and has clumpy, asymmetric structure. However, a major role is also played by the constraints from the present X-ray instruments. While $1 / t$ for a steady wind has been derived for a total X-ray luminosity (Fransson et al. 1996), current telescopes measure $\mathrm{X}$-ray flux in a narrow $\mathrm{X}$-ray range. This could give deviation from the expected luminosity profile, as well as may result in erroneous estimates of the parameters. It is crucial to have a wind band X-ray coverage to truly understand the nature of the X-ray emission. For example, in SN 2010jl, the joint XMM-Newton and $N u S T A R$ spectrum (see Fig. 3) covering energy range $0.3-80 \mathrm{keV}$ pinpointed the thermal $\mathrm{X}$-ray emission temperature to be $\sim 20 \mathrm{keV}$ and established forward shock origin of the X-ray emission (Ofek et al. 2014, Chandra et al. 2015). This is the first time the (forward) shock temperature was observationally accurately measured in a SN.

\subsection{Absorption of radio emission}

On one hand high density CSM means efficient production of synchrotron radio emission, on the other hand higher density also translates into higher absorption. In classic core collapse $\mathrm{SNe}$, the radio emission is absorbed either by the ionized CS medium (free-free 

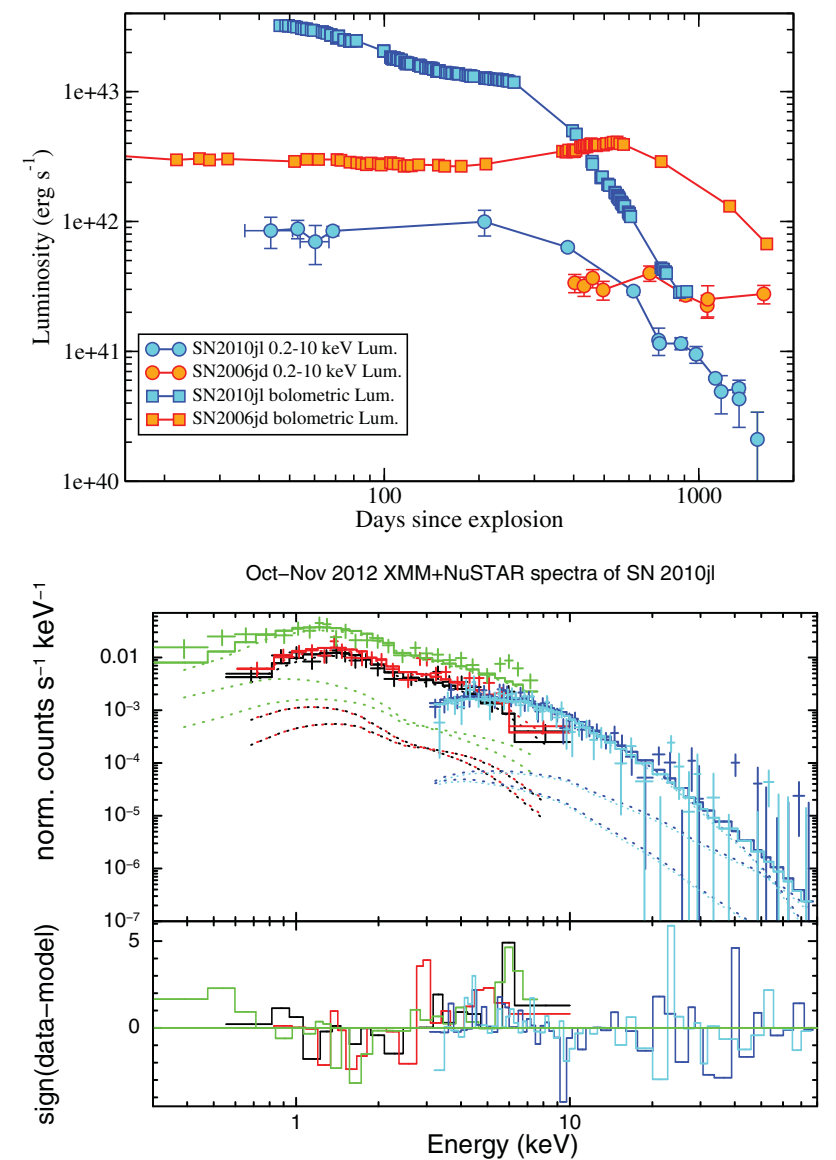

Figure 3. Upper panel: The blue curves are the $0.2-10 \mathrm{keV}$ X-ray luminosity evolution of two SNe IIn 2006jd and 2010jl. None of them follow $\sim 1 / t$ dependence. Lower panel: Joint $N u S T A R$ and XMM-Newton spectrum of the SN 2010jl covering energy range $0.3-80 \mathrm{keV}$ (reproduced from Chandra et al. (2015)).

absorption (FFA)) or by the in situ relativistic electrons (synchrotron self-absorption (SSA)). Observations when the SN is rising in the light curve evolution can disentangle the underlying absorption mechanism. The role of absorption mechanisms is different for different SNe (Chevalier et al. 2003). SNe IIn are expected to have external FFA due to their high density. However, in SNe IIn due to high density, radiative cooling shell is formed in the shocked region. The mixing of the cool gas in the forward shocked shell may cause some SNe IIn to undergo internal FFA. This indeed has been seen in SN 2006jd (Fig. 4 and Chandra et al. 2012), SN 1986J (Weiler et al. 1990) and SN 1988Z (van Dyk et al. 1993, Williams et al. 2002). Chandra et al. (2012a) have shown that modest amount of cool gas $\left(\sim 10^{4} \mathrm{~K}\right)$ i.e. $\sim 10^{-8} \mathrm{M}_{\odot}$ can explain internal absorption in SN 2006jd.

The radio observations of SNe IIn also indicate that most of the SNe IIn are late radio emitters (e.g. Chandra et al. 2015). This could be partially due to the high absorption as well as partially due to observational biases. Some of the radio emitting SNe IIn were either discovered late or classified late, such as SN 1995N, 1986J, and 2006jd. SNe IIn which were discovered soon after the explosion, most of them did not have early radio observations within a month, except for SN 2009ip (Margutti et al. 2014). SN 2009ip was 


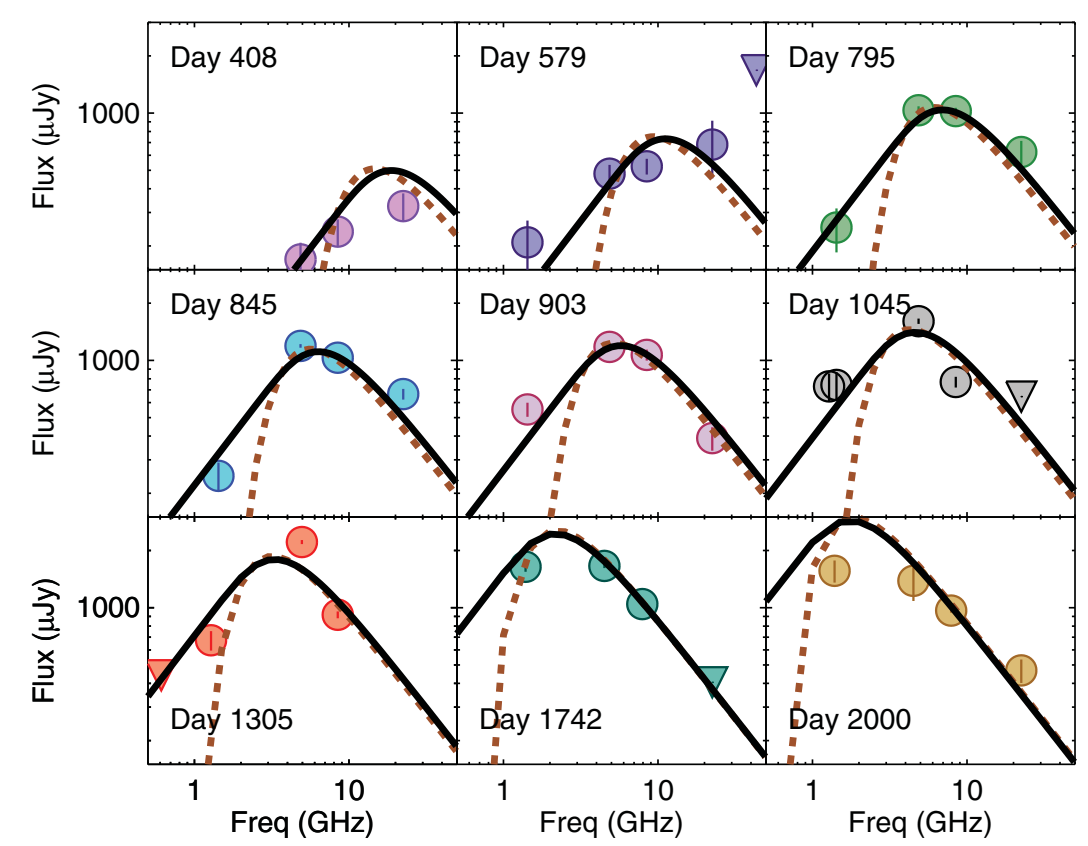

Figure 4. Spectra of SN 2006jd reproduced from Chandra et al. (2012a). Here internal free-free absorption (black solid lines) best fit the observed absorption.

detected in radio bands early on but faded below detection within a few tens of days. However, SN 2009ip was a peculiar SN with poorly understood explosion mechanism. In contrast the observations of SN 2010jl started by day 45 but the first detection happened only after 500 days (Chandra et al. 2015), clearly showcasing the efficient absorption of radio emission at early epochs.

The late radio turn on may also explain the overall low detection rates of SNe IIn. If a $\mathrm{SN}$ is not bright in radio bands in a first few epochs, the observational campaign for that SN is usually over. So even if a SN IIn was a potential radio emitter at later epochs, it would be missed in radio bands. van Dyk et al. (1996) observed 10 SNe IIn at an age of a few hundred days and still did not detect any; they set upper limits of $150-250$ $\mu \mathrm{Jy}$. However, the current telescopes are able to reach at least an order of magnitude better sensitivity. In addition, the current upgraded VLA is extremely sensitive at higher frequencies where the radio absorption effects vanish much sooner $\left(\tau_{\lambda} \propto \lambda^{2}\right)$. Strategic study of SNe IIn at higher frequencies at early times to low frequencies at late epochs is likely to result in the most complete sample of radio supernovae (Pérez-Torres et al. 2014, Wang et al., 2015). SKA will be ideally suited for such studies at low frequencies. With an improved sensitivity level of $1 \mu \mathrm{Jy}$ with Square Kilometre Array (SKA) one can detect the brightest of radio SNe, such as the Type IIn SN $1988 \mathrm{Z}$ up to redshift of $\mathrm{z}=1$ (Pérez-Torres et al. 2014),

\subsection{Evolution of column density}

SN 2010jl is the best studied X-ray supernova, where X-ray observations covered a period up to day 1500 with Chandra, XMM-Newton, NuSTAR, and Swift X-ray Telescope. This gave us opportunity to trace the evolution of column density from 40 to 1500 days. We witnessed three orders of magnitude change in column density over the span of X-ray observations (Chandra et al. 2012b, Chandra et al. 2015). At first epoch the column density associated with the SN was $N_{H}=10^{24} \mathrm{~cm}^{-2}$ (for a metallicity of 0.3 solar, Stoll 


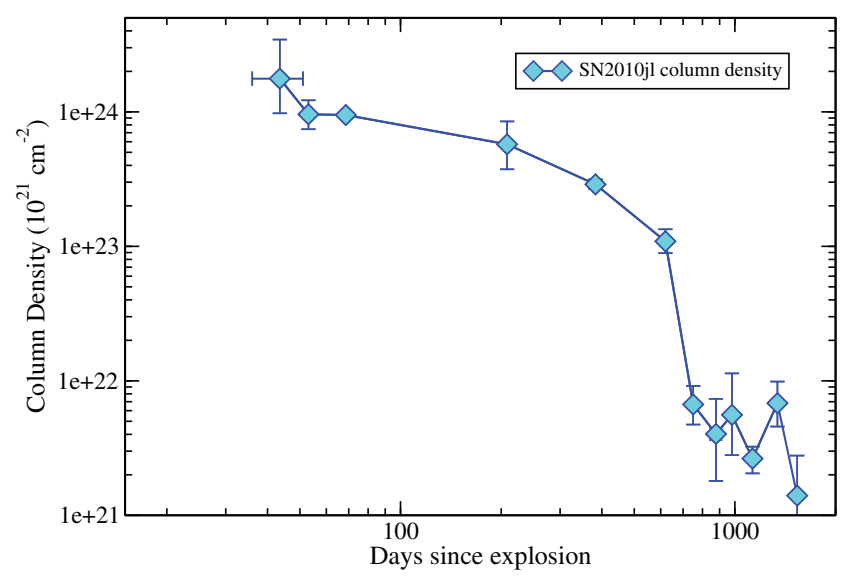

Figure 5. The column density evolution in SN 2010jl. The figure is reproduced from Chandra et al. (2015).

et al. 2011), 3000 times higher than the Galactic column density, which declined slowly by an order of magnitude upto day $\sim 650$. Afterwards followed by a sharp decline, it settled to 10 times the Galactic value. The evolution of column density from Chandra et al. (2015) have been reproduced in Fig. 5.

The higher column density observed in the X-ray observations for SN 2010jl is not associated with the high host galaxy extinction. This indicates that the higher column density is due to the mass loss near the forward shock and thus is arising from the CSM. This was consistent with the variability of the column density too. This was the first time that external circumstellar X-ray absorption had been clearly observed in a SN and enabled us to trace the precise mass loss evolution history of the star.

\subsection{Episodic mass loss?}

It is becoming increasingly evident that the mass loss rate from some SNe IIn may not be steady. Unfortunately it is difficult to disentangle this effect because SNe IIn likely to remain highly absorbed for a long duration. By the time they become optically thin in radio bands, the strength of synchrotron emission is rather weak to see any such effects of mass loss. In addition, one needs a long term follow up to see the episodic mass loss. In X-ray observations, due to the lack of well sampled data, such trends are easy to miss. Yet, it seems that some SNe IIn seem to suggest episodic mass loss rate.

In Fig. 6, X-ray and radio light curves are plotted for a Type IIn SN 1995N (Chandra et al. 2009). The X-ray bands were chosen to mimic the ROSAT and ASCA bands. The radio data is at the four representative VLA frequencies. Around day $\sim 1300$ onwards, bumps in the X-ray and radio light curves are seen. The long gap in the X-ray light curve does not allow to witness the evolution of this bump, however, in the radio data one can clearly see this effect. Due to the sensitivity limitation of the telescopes and lack of long term follow up, its not possible to explore the further bumps in time, if any. In SN 2006jd, one can also see a hint of such bumps near simultaneously in both X-ray and radio bands (Chandra et al. 2012a). The radio emission is too weak in SN 2010jl to note any such bumps. However, X-ray data seems to suggest a steady decline followed by a sudden decline settling down to steady decline again at $\sim 1000$ days. (Chandra et al. 2015). These trends could be suggestive of the episodic mass loss rates in some SNe IIn and may support the LBV scenario. However, further study and much longer follow ups are needed. 

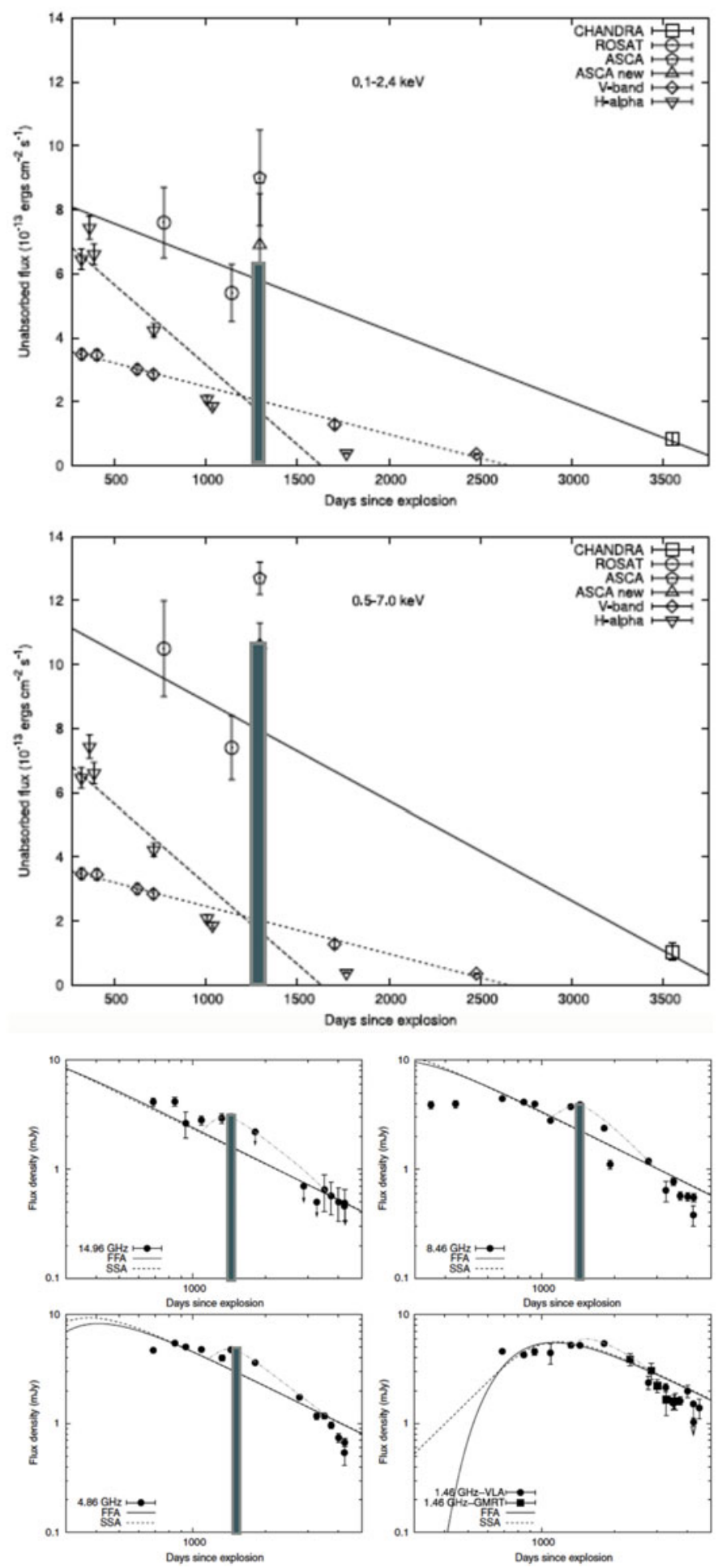

Figure 6. Radio and X-ray light curves of SN 1995N (Chandra et al. 2009). A hint of enhanced emission bump in radio and X-ray bands of a Type IIN SN $1995 \mathrm{~N}$ around $\sim 1300$ days. 


\subsection{Asymmetry in the explosion}

In optical bands, there has been various pieces of evidence suggesting asymmetry in the explosion. Polarization observations have indicated large-scale asymmetry, e.g., SN 1997eg (Hoffman et al. 2008), SN 1998S (Leonard et al. 2000), SN 2010jl (Patat et al. 2011). Many of the models explaining SNe IIn also require bipolar wind or disk like geometry (see Smith (2016a) for a review). In some SNe IIn, radio and X-ray interpretation of the data too seem to favour the asymmetric models.

SN 2010jl early optical spectra showed the presence of broad emission lines (Smith et al. 2012, Fransson et al. 2014, Ofek et al. 2014), explained by an electron scattering optical depth $>1-3$, i.e., a column density $N_{H} \geqslant 3 \times 10^{24} \mathrm{~cm}^{-2}$, comparable with the column density seen in the first X-ray observations (Chandra et al. 2015). At later epochs $\left(t>70\right.$ day) X-rays column density declined to $N_{H}<3 \times 10^{24} \mathrm{~cm}^{-2}$. These constraints are difficult to reconcile with a spherical model of column density $3 \times 10^{24}$ $\mathrm{cm}^{-2}$. This may suggest that the X-rays escape the interaction region, avoiding the high column density CSM. This fits well in a scenario for the CSM having a bipolar geometry.

In SN 2006jd, it is difficult to reconcile the X-ray and radio data under spherical models (Chandra et al. 2012a) too. The column density of the matter to the X-ray emission was $\sim 50$ times smaller than what was needed to produce the X-ray luminosity. The absorption column depends on the CSM wind density as $\propto \rho_{w}$ and the X-ray luminosity as $\propto \rho_{w}^{2}$. While clumpiness may explain this scenario, it lead to inconsistent shock velocity derived from the observed X-ray spectrum. The situation was explained if the CSM clouds interact primarily with the cool dense shell. This suggested that there is a global asymmetry in the CSM gas distribution that allowed a low column density in one direction, unaffected by the dense interaction taking place over much of the rest of the solid angle as viewed from the SN. This would also explain low column density to the radio emission too, because both radio and X-ray are from the same region. The lack of external FFA could also be explained in this model (Chandra et al. 2012a).

\section{Summary}

In this conference proceeding, we have summarized our current understanding of SNe IIn, mainly from the radio and X-ray observations. While X-ray luminosities seem to be occupying systematically high values, radio luminosities do not stand out. Due to their extreme densities, SNe IIn suffer absorption for a longer time and have tendency to turn up late in radio bands. Many SNe IIn radio spectra seem to be consistent with the internal free-free absorption, an effect negligible in other core collapse SNe. This is due to the formation of radiative shell. Cool gas $\left(10^{4} \mathrm{~K}\right)$ mixed into the hot shocked gas is efficient in doing the internal FFA. To disentangle the true nature of radio emission, multiple frequency sensitive radio observations spanning a large time span are needed.

The X-ray emission in SNe IIn is usually dominated by the forward shock, as radiative reverse shock emission is absorbed by the cool dense shell formed between the two shocks. None of the SNe IIn seem to follow X-ray luminosity evolution of $L \propto 1 / t$ for steady spherical wind. Before getting into the physical reasons, one needs to understand telescope biases. Most of the X-ray telescopes works in narrow X-ray filters hence one gets spectral $\mathrm{X}$-ray luminosity, whereas the above evolution is valid for the total X-ray luminosity. $N u S T A R$ combined with other X-ray telescopes like $X$ MM-Newton is a very promising telescope to obtain total X-ray luminosity. In addition, it is also emerging that the wind density profiles are far from standard $1 / R^{2}$. There is certainly a hint of episodic mass ejection in some SNe IIn. SN 2010jl is an ideal example to follow the mass loss evolution 
due to its excellent X-ray coverage. Finally we note that since more than $50 \%$ O stars are in binary systems (Sana et al. 2012), one does need to take into account SN evolution under binary scenario. In addition disk like and bipolar asymmetries cannot be ignored while studying SNe IIn.

$\mathrm{PC}$ acknowledges support from the Department of Science and Technology via SwaranaJayanti Fellowship award (file no. DST/SJF/PSA-01/2014-15).

\section{References}

Anderson, J. P., Habergham, S. M., James, P. A., \& Hamuy, M. 2012, MNRAS, 424, 1372

Bilinski, C., Smith, N., Li, W., et al. 2015, MNRAS, 450, 246

Chandra, P., Stockdale, C. J., Chevalier, R. A., et al. 2009, ApJ, 690, 1839

Chandra, P., Chevalier, R. A., Chugai, N., et al. 2012, ApJ, 755, 110

Chandra, P., Chevalier, R. A., Irwin, C. M., et al. 2012, ApJ Letters, 750, L2

Chandra, P., Chevalier, R. A., Chugai, N., Fransson, C., \& Soderberg, A. M. 2015, ApJ, 810, 32

Chevalier, R. A. \& Fransson, C. 2003, Supernovae and Gamma-Ray Bursters, Edited by K. Weiler., Lecture Notes in Physics, 598, 171

Chevalier, R. A. 2012, ApJ Letters, 752, L2

Chevalier, R. A. \& Fransson, C. 2016, arXiv:1612.07459

Chugai, N. N. 1990, Soviet Astron. (Letters), 16, 457

Chugai, N. N. \& Chevalier, R. A. 2006, ApJ, 641, 1051

Cooke, J., Sullivan, M., Barton, E. J., et al. 2009, Nature, 460, 237

Dwarkadas, V. V. \& Gruszko, J. 2012, MNRAS, 419, 1515

Dwarkadas, V. V., Romero-Cañizales, C., Reddy, R., \& Bauer, F. E. 2016, MNRAS, 462, 1101

Fox, O. D., Van Dyk, S. D., Dwek, E., et al. 2017, ApJ, 836, 222

Fransson, C., Lundqvist, P., \& Chevalier, R. A. 1996, ApJ, 461, 993

Fransson, C., Ergon, M., Challis, P. J., et al. 2014, ApJ, 797, 118

Gal-Yam, A., Leonard, D. C., Fox, D. B., et al. 2007, ApJ, 656, 372

Hoffman, J. L., Leonard, D. C., Chornock, R., et al. 2008, ApJ, 688, 1186-1209

Leonard, D. C., Filippenko, A. V., Barth, A. J., \& Matheson, T. 2000, ApJ, 536, 239

Margutti, R., Milisavljevic, D., Soderberg, A. M., et al. 2014, ApJ , 780, 21

Mauerhan, J. C., Smith, N., Filippenko, A. V., et al. 2013, MNRAS, 430, 1801

Milisavljevic, D., Margutti, R., Kamble, A., et al. 2015, ApJ, 815, 120

Nymark, T. K., Fransson, C., \& Kozma, C. 2006, A\& A, 449, 171

Ofek, E. O., Sullivan, M., Shaviv, N. J., et al. 2014a, ApJ, 789, 104

Ofek, E. O., Zoglauer, A., Boggs, S. E., et al. 2014b, ApJ, 781, 42

Patat, F., Taubenberger, S., Benetti, S., Pastorello, A., \& Harutyunyan, A. 2011, A\& A, 527, L6

Pérez-Torres, M. A., Lundqvist, P., Beswick, R. J., et al. 2014, ApJ, 792, 38

Ross, M. \& Dwarkadas, V. 2017, arXiv:1704.05866

Sana, H., de Mink, S. E., de Koter, A., et al. 2012, Science, 337, 444

Smith, N., Li, W., Foley, R. J., et al. 2007, ApJ, 666, 1116

Smith, N., Li, W., Miller, A. A., et al. 2011, ApJ, 732, 63

Smith, N., Silverman, J. M., Filippenko, A. V., et al. 2012, AJ, 143, 17

Smith, N. \& Tombleson, R. 2015, MNRAS, 447, 598

Smith, N. 2016a, arXiv:1612.02006

Smith, N., Andrews, J. E., \& Mauerhan, J. C. 2016b, MNRAS, 463, 2904

Stoll, R., Prieto, J. L., Stanek, K. Z., et al. 2011, ApJ,, 730, 34

Taddia, F., Stritzinger, M. D., Sollerman, J., et al. 2013, A\& A, 555, A10

van Dyk, S. D., Weiler, K. W., Sramek, R. A., \& Panagia, N. 1993, ApJ Letters, 419, L69

van Dyk, S. D., Weiler, K. W., Sramek, R. A., et al. 1996, AJ, 111, 1271

Wang, L., Cui, X., Zhu, H., \& Tian, W. 2015, Advancing Astrophysics with the Square Kilometre Array (AASKA14), 64

Weiler, K. W., Panagia, N., \& Sramek, R. A. 1990, ApJ, 364, 611

Williams, C. L., Panagia, N., Van Dyk, S. D., et al. 2002, ApJ, 581, 396 